\section{The therapy of gefitinib towards breast cancer partially through reversing} breast cancer biomarker arginine

Geng Dongmei\#, Sun Dengjun\#, Zhang Liangming, Zhang Weiwei

Department of Medical Oncology, Affiliated Yuhuangding Hospital, Medical College of Qingdao University, 20 Yuhuangding East Road, Zhifu District, Yantai, Shandong 264000, China.

\# These two authors equally contributed to this work.

\section{Abstract}

Background: Breast cancer remains the leading reason of cancer death among women worldwide, and gefitinib is the efficient drug for breast cancer.

Aims: To use targeted metabolomics method to elucidate the therapeutic mechanism of gefitinib through profiling the mino acids.

Methods: Healthy women $(n=56)$ and women with breast cancer $(n=60)$ were enrolled in Affiliated Yuhuangding hospital, medical college of Qingdao University from 2012-2014. API 3200 triple quadrupole mass spectrometer was used to analyze the serum samples.

Results: The concentration of amino acids was compared between healthy women and women with breast cancers. Compared with the healthy women, the concentration of arginine in breast cancer women significantly decreased $(\mathrm{p}<0.0001)$. To show the representative capability of arginine towards the pathogenesis of breast cancers, the receiver operating charac teristic $(\mathrm{ROC})$ curve was drawn, and the area under the curve (AUC) was calculated to be $0.96 \pm 0.02$, indicating the high predictive capability of arginine for breast cancer. The reversing ability of gefitinb towards the level of arginine was figh ( determined, and 1 month treatment of gefitinib $(500 \mathrm{mg} / \mathrm{day})$ significantly reversed the arginine level of breast cancer patients $(\mathrm{p}<0.0001)$

Conclusion: The therapy of gefitinib towards breast cancer through reversing breast cancer biomarker arginine was demonstrated.

Keywords: Breast cancer, arginine, metabolomics, gefitinib

DOI: http://dx.doi.org/10.4314/ahs.v15i2.36

\section{Introduction}

Breast cancer remains the leading reason of cancer death among women worldwide ${ }^{1}$. There are many risk factors for breast cancers, such as obesity, lack of physical exercise, and drinking alcohol. Many drugs have been used, are being developed, or exert the therapeutic potential towards breast cancers, such as paclitaxel, doxorubicin, fluorouracil, Everolimus, and anastrozole(http://www. cancer.gov/cancertopics/druginfo/breastcancer).

\section{Corresponding author: \\ Zhang Weiwei, \\ Department of Medical Oncology, \\ Affiliated Yuhuangding Hospital, \\ Medical College of Qingdao University, \\ 20 Yuhuangding East Road, Zhifu District, \\ Yantai, Shandong 264000, China.}

E-mail: Ouuu5987@126.com
Gefitinib, under the trade name Iressa, marketed by AstraZeneca and Teva, is a drug employed to treat breast cancer ${ }^{2}$. Additionally, gefitinib can also be employed to treat many other tumors, such as lung cancer. Gefitinib inhibits EGFR tyrosine kinase by binding to the adenosine triphosphate (ATP)-binding site of the enzyme. Therefore, EGFR tyrosine kinase has been widely accepted as the major target for gefitinib in breast cance treatment. However, a drug always has multiple therapeutic targets and mechanisms, and combination of multiple target pathways can facilitate the explanation of therapeutic mechanism. Therefore, searching new therapeutic mechanism of gefitinib towards breast cancer will provide the opportunity to completely explain the therapeutic mechanism of gefitinib towards breast cancer.

Metabolomics is the scientific study of chemical processes involving small molecules metabolites ${ }^{3}$. Metabolomics study has played a key role in elucidating the mechanism of various cancers. Metabolomics study of colon cancers showed that the elevation of level of taurine, isoglutamine, choline, lactate, phenylalanine and tyrosine, and the reduction of lipids and triglycerides is closely related with the pathogenesis of colon cancer ${ }^{4}$. For breast cancer, the alteration of histidine, acetoacetate, glycerol, pyruvate, glycoproteins (N-acetyl), mannose, glutamate and phenylalanine has been demonstrated to be the key factor ${ }^{5}$.

The present study aims to determine the therapeutic mechanism of gefitinib using targeted metabolomics.

\section{Materials and methods}

\section{The statement of Ethics}

Ethical approval for this study was granted from affiliated Yuhuangding hospital, and all enrolled women in this study gave the written informed consent.

\section{Study Population}

Healthy women $(\mathrm{n}=56)$ and women with breast cance $(\mathrm{n}=60)$ were enrolled in Affiliated Yuhuangding hospital, medical college of Qingdao University from 20122014. The serum was taken before and after treatment with $500 \mathrm{mg}$ /day for 1 months through centrifugation (the speed $8,000 \mathrm{~g}$ ) of blood.

Targeted metabolomics analysis of amino acids components

The aliquots $(10 \mathrm{uL})$ of serum were dropped on the fil- Result

ter paper, and then filter paper was placed into 96-well The concentration of amino acids was firstly compared polypropylene micro titer plates. $100 \mu \mathrm{L}$ of methanol between healthy women and women with breast cansolution containing isotope labeled amino acid internal cers. As shown in Figure 1, the concentration of arginine was $14.7 \pm 0.9 \mathrm{uM}$

Figure 1 Arginine significantly decreased in pre-treatment patients with breast cancer. The concentration of arginine in health volunteers $(\mathrm{n}=56)$ and patients $(\mathrm{n}=60)$ was given in Figure. $* * * *, \mathrm{p}<0.0001$.

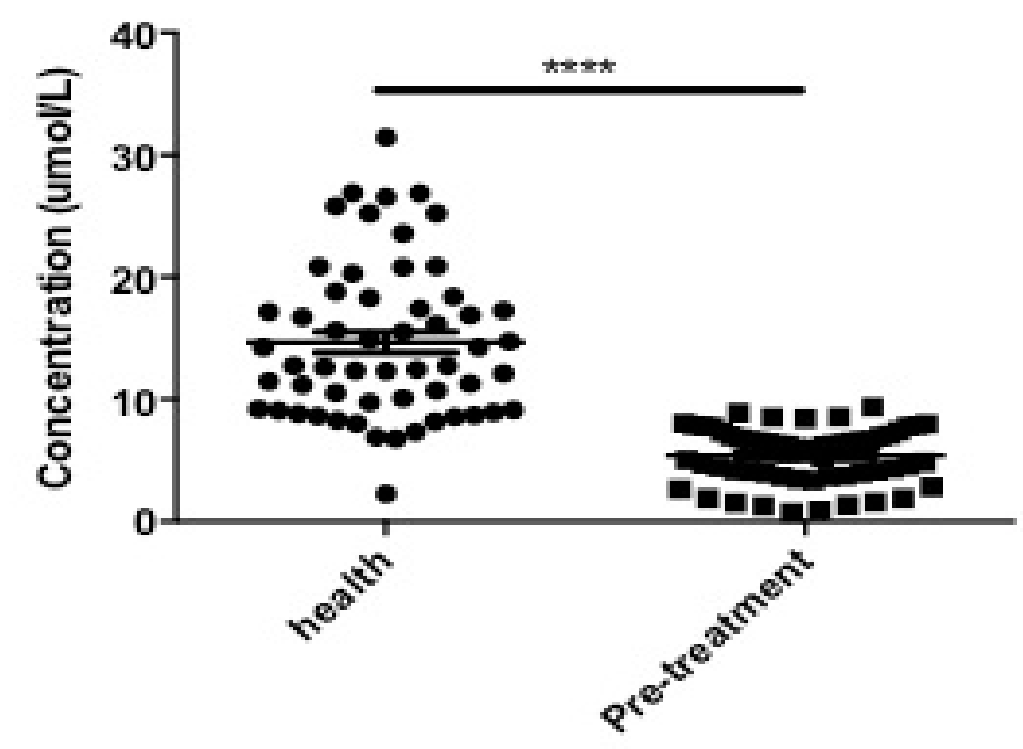


Compared with the healthy women, the concentra- genesis of breast cancers, the receiver operating chardecreased (value $=5.4 \pm 0.3, p<00001$ ). To show the the curve $(\mathrm{AUC})$ was calculated to be $0.96 \pm 0.02$, in representative capability of arginine towards the patho- dicating the high predictive capability of arginine for breast cancer (Figure 2).

Figure 2 Receiver operating characteristic (ROC) curve to demonstrate the sensitivity and specificity for Arginine as the prediction biomarker.

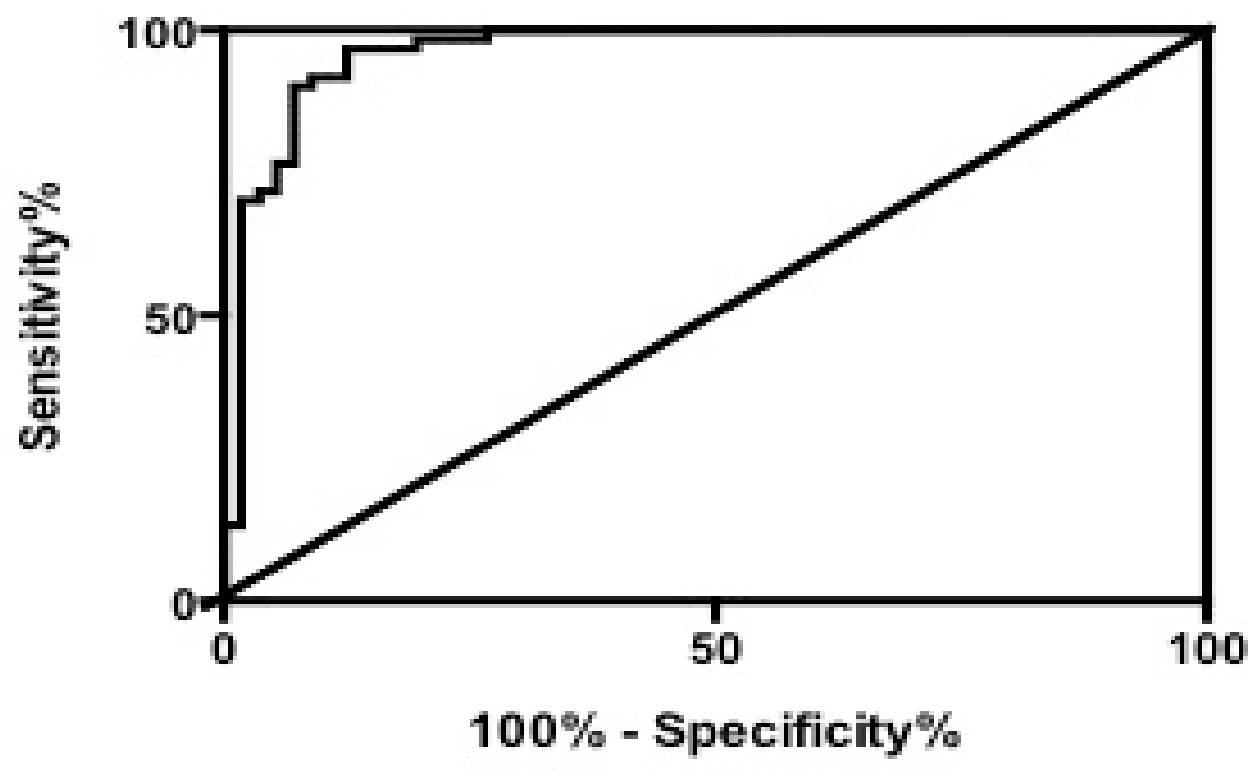

The reversing ability of gefitinib towards the level of the arginine level of breast cancer patients $(p<0.0001)$ arginine was further determined, and 1 month treat- (Figure 3). Other amino acids did not changed among ment of gefitinib $(500 \mathrm{mg} /$ day) significantly reversed these three groups (data not shown).

Figure 3 Comparison of arginine level in serum from pre-treatment and after-treatment patients $(\mathrm{n}=60)$. ****, $\mathrm{p}<0.0001$.

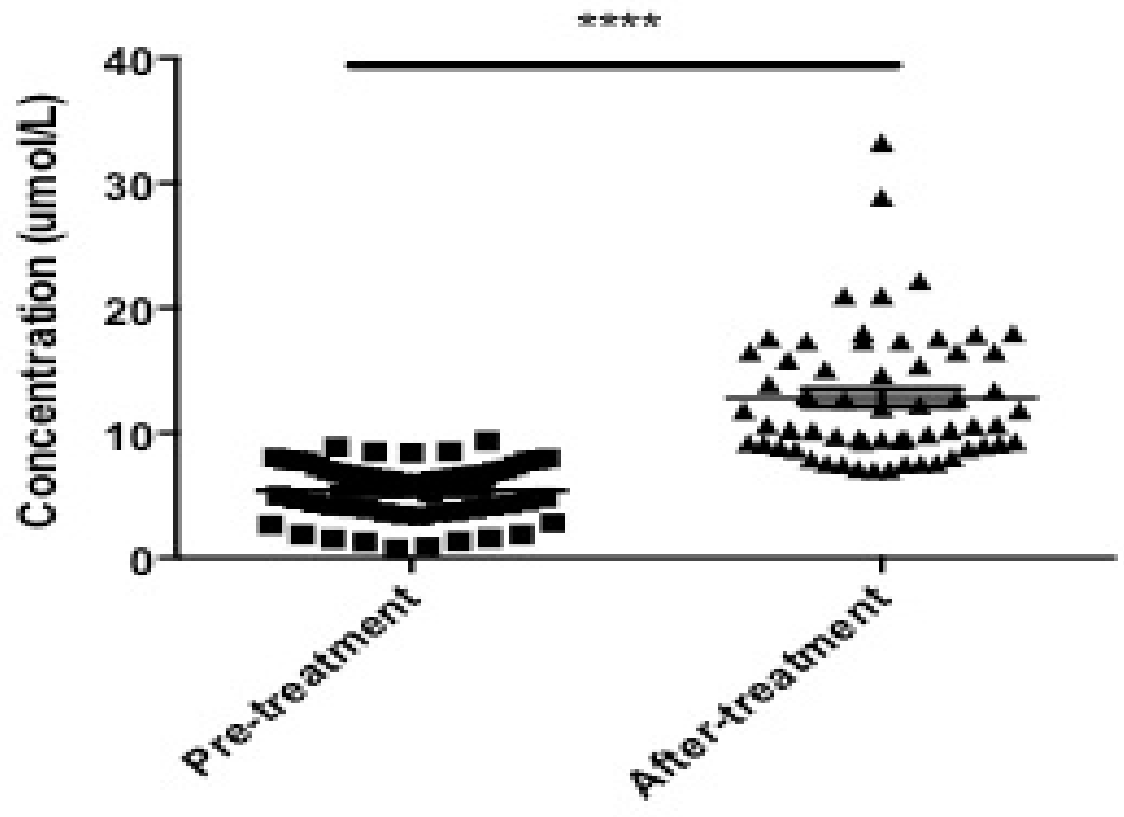

Amino acids are organic compounds that combine to form proteins, and amino acids and proteins are the building blocks of life. Additionally, amino acids are important energy source for the body ${ }^{6}$. The present study showed that arginine significantly decreased in breast cancer patients in comparison with healthy volunteers. The decrease of aroinine in serum indicated possible elevation of arginine in tumor tissues. Previous literature showed that arginine starvation could protect breast cancer cells through impairing mitochondrial respiratory function ${ }^{7}$. Therefore, gefitinib-induced elevation of arginine in serum for gefitinib-treated patients might indicate the possible therapy role through decreasing the arginine level in tumor tissues. .

2. Kalykaki A, Agelaki S, Kallergi G, Xyrafas A, Mav roudis D, Georgoulias V. Elimination of EGFR-expressing circulating tumor cells in patients with metastatic breast cancer treated with gefitinib. Cancer Chemother. Pharmacol. 2014; 73(4):685-693.

3. Fang ZZ, Gonzalez FJ. LC-MS-based metabolomics: an update. Arch. Toxicol. 2014; 88(8):1491-1502.

4. Jimenez B, Mirnezami R, Kinross J, Cloarec O, Keun He, Holmes E, et al. $1 \mathrm{H}$ HR-MAS NMR spectroscopy of tumor-induced local metabolic "field-effects" enables colorectal cancer staging and prognostication. J. Proteome Res. 2013; 12(2):959-968.

5. Bezabeh T, Ijare OB, Nikulin AE, Somorjai RL, It should be noted previous literature showed that tryp- Smith IC. MRS-based Metabolomics in Cancer Retophan is the key native marker in cells to determine the level of metastasis competence in breast cell lines using native fluorescence spectroscopy ${ }^{8}$. However, the present study did not detect the alteration of tryptophan in breast cancers, and the reversing effect of gefitinib towards tryptophan. The reason might be the number of patients is not enough, and maybe the in vitro results are not easily translated into in vivo situation.

search. Magn. Reson. Insights 2014; 7:1-14.

6. Zhou J, Yan W, Hu G, Shen B. Amino Acid network for the discrimination of native protein structures from decoys. Curr. Protein Pept. Sci. 2014; 15(6):522-528.

7. Qiu F, Chen YR, Liu X, Chu CY, Shen LJ, Xu J, et al. Arginine starvation impairs mitochondrial respiratory function in ASS1-deficient breast cancer cells. Sci. Signal. 2014; 7(319):ra31.

In conclusion, the therapy of Gefitinib towards breast 8. Zhang L, Pu Y, Xue J, Pratavieira S, Xu B, Achilefu cancer through reversing breast cancer biomarker arginine was demonstrated.

$\mathrm{S}$, et al. Tryptophan as the fingerprint for distinguishing aggressiveness among breast cancer cell lines using native fluorescence spectroscopy. J. Biomed. Opt. 2014; 19(3):37005. 